\title{
Creating and probing coherent atomic states
}

\author{
C. O. Reinhold and J. Burgdörfer \\ Sind \\ Physics Division, Oak Ridge National Laboratory, Oak Ridge, TN 37831-6379 and \\ Dept. of Physics and Astronomy, University of Tennessee, Knoxville TN 37996-1200.
}

\author{
M. T. Frey and F. B. Dunning
}

Dept. of Physics and the Rice Quantum Institute, Rice University, 6100 S. Main Street, Houston, TX

77005-1892

We present a brief review of recent experimental and theoretical time resolved studies of the evolution of atomic wavepackets. In particular, wavepackets comprising a superposition of very-high-lying Rydberg states which are created either using a short half-cycle pulse (HCP) or by rapid application of a DC field. The properties of the wavepackets are probed using a second HCP that is applied following a variable time delay and ionizes a fraction of the atoms, much like a passing-by ion in atomic collisions. 


\section{INTRODUCTION}

The interest in creating coherent atomic states dates back to early attempts by Schrödinger [1] to construct a non-dispersive minimum uncertainty wavepacket similar to coherent oscillator eigenstates. The latter follow Newton's laws of classical mechanics while the width in position and momentum remains at the minimum consistent with the Heisenberg uncertainty principle. The early attempts failed for electronic states in atoms because of the non-equidistant spacing of energy levels which leads to rapid dephasing. Subsequently, the production a of more general class of wavepackets have been studied which consist of a dispersive coherent superposition of atomic states and do not satisfy the minimum uncertainty condition. Examples of signatures of coherences associated with the electronic time evolution in atomic collisions include, from very old ones to very recent ones: Stückelberg oscillations [2], Stark beats [3], and swapping oscillations [4]. Only in recent years it has become possible to generate transient quasi-classical wavepackets which remain well localized for a long period of time. This has been accomplished by using Rydberg states with large principal quantum numbers, $n$, for which the deviation from equidistant levels decreases as $n^{-1}$ and for which the wavepacket evolution resembles the one for the harmonic oscillator. These so-called Rydberg wavepackets are nonstationary states consisting of a coherent superposition of nearby atomic eigenstates.

In the last decade there has been increasing interest in the time-resolved study of atomic wavepackets by means of newly developed short electromagnetic pulses. They frequently display novel dynamical behavior that mimics the classical motion of the excited electron thereby providing a bridge between quantum and classical physics. Rydberg wavepackets were first created by photoexcitation of groundstate atoms using ultrashort laser pulses whose bandwidth exceeded the level spacing in the final Rydberg manifold [5-8]. Since photon absorption occurs when the electron is close to the core ion, the wavepacket is initially strongly localized in the vicinity of the core. As time advances, the wavepacket expands and contracts radially very much like a classical particle in a Kepler orbit. This behavior is then monitored using a second ultrafast probe pulse which ionizes the atom. 


\section{DISCLAIMER}

This report was prepared as an account of work sponsored by an agency of the United States Government. Neither the United States Government nor any agency thereof, nor any of their employees, make any warranty, express or implied, or assumes any legal liability or responsibility for the accuracy, completeness, or usefulness of any information, apparatus, product, or process disclosed, or represents that its use would not infringe privately owned rights. Reference herein to any specific commercial product, process, or service by trade name, trademark, manufacturer, or otherwise does not necessarily constitute or imply its endorsement, recommendation, or favoring by the United States Government or any agency thereof. The views and opinions of authors expressed herein do not necessarily state or reflect those of the United States Government or any agency thereof. 


\section{DISCLAIMIER}

Portions of this document may be illegible in electronic image products. Images are produced from the best available original document. 
Here we discuss a more recent approach to the generation of wavepackets starting with a stationary Rydberg atom and using the so-called "half-cycle pulses" (HCPs) [9-20]. In contrast to short laser pulses which extend over several optical cycles, HCPs are characterized by a strong unidirectional electric field $\vec{F}(t)$ confined to a short time interval, $T_{p}$, corresponding to only a fraction of a cycle. These characteristics make HCPs very similar to the transverse electric field pulse generated by the passing-by projectile in a fast ion-atom collision. Thus, the study of the dynamics of Rydberg atoms subject to HCPs provides the building block of problems of practical importance such us the transport of fast ions and atoms through solids [21]. Recent experiments have reached the regime in which the duration of the pulses, $T_{p}$, is shorter than the classical electron orbital period, $T_{n_{i}}=2 \pi n_{i}^{3}$, associated with the stationary initial state with principal quantum number $n_{i}$ (atomic units are used throughout). This has been accomplished using subpicosecond pulses and moderately low $n_{i} \sim 30$ Rydberg levels [9] or, alternatively, using nanosecond pulses and very high $n_{i} \sim 400$ states [10]. In this work we focus on the latter.

The effect of a HCP on an atom differs significantly from that generated by an ultrashort laser pulse because the integral of the applied electric field is finite. In the limit of ultrashort HCPs, $T_{0}=T_{p} / T_{n_{i}} \ll 1$, the HCP simply delivers a "kick" or impulsive momentum transfer

$$
\Delta \vec{p}=-\int_{-\infty}^{\infty} \vec{F}(t) d t
$$

to the electron [11]. This is, in fact, the result of the sudden or impulse approximation, well known in atomic collisions. The momentum transfer leads directly to excitation to higher lying states and can, for sufficiently strong pulses, even induce ionization (i.e. for momentum transfers of the order of the orbital velocity of the electron in the atom: $\left.\Delta p \gtrsim p_{n_{i}}=n_{i}^{-1}\right)$. In the ultrashort pulse limit, the energy transferred to a classical electron with momentum $\vec{p}$ is given by

$$
\Delta E=\frac{(\Delta p)^{2}}{2}+\vec{p} \cdot \Delta \vec{p}
$$

Depending on the projection of the electronic momentum in the direction of the HCP, the energy transfer 
can be positive or negative and can lead to ionization. Therefore, HCPs provide a tool to study the time-evolution of the momentum of the electron.

\section{PRODUCING AND PROBING WAVEPACKETS}

Consider an electron whose dynamics is governed by a time-independent Hamiltonian $H_{0}$ with orthonornal eigenvectors and eigenenergies $\left|\chi_{\alpha}\right\rangle$ and $\epsilon_{\alpha}$, respectively. The wavefunction of the electron evolves in time according to

$$
|\Psi(t)\rangle=\sum_{\alpha} e^{-i \epsilon_{\alpha} t}\left\langle\chi_{\alpha} \mid \Psi(0)\right\rangle\left|\chi_{\alpha}\right\rangle
$$

which is a "fully coherent" wavepacket (i.e. a pure state at each instant of time). The mean value of an observable $O$ is given by

$$
\langle O\rangle=\sum_{\alpha, \beta} e^{-i\left(\epsilon_{\alpha}-\epsilon_{\beta}\right) t}\left\langle\chi_{\alpha} \mid \Psi(0)\right\rangle\left\langle\Psi(0) \mid \chi_{\beta}\right\rangle\left\langle\chi_{\beta}|O| \chi_{\alpha}\right\rangle
$$

and oscillates in time with frequencies $\omega_{\alpha, \beta}=\epsilon_{\alpha}-\epsilon_{\beta}$, usually referred to as "quantum beats".

A wavepacket can be created through a time-dependent perturbation which acts as a "pump". Subsequently, a quantum beating frequency $\omega_{k, j}$ can be observed using another time-dependent perturbation (a "probe") with a time duration $T_{p}$ such that $T_{p} \lesssim\left(2 \pi / \omega_{\alpha, \beta}\right)$. Figure 1 shows two possible schemes for producing and probing wavepackets from an initially stationary Rydberg state using a time-dependent unidirectional electric field $F(t)$. The field profiles in the figure are actual experimental data. In the scheme of Fig. 1(a), both the pump and the probe are short HCPs. The time evolution of the wavepacket can be studied by analyzing the survival probability of Rydberg atoms as a function of the time delay between the two HCPs. In this case, $H_{0}=H_{a t}$ (i.e. the free atomic Hamiltonian).

The pump in Fig. 1(b) is given by a "field step" which, in fact, corresponds to the rapid rise of a very 
long "rectangular" HCP. This field step establishes a "DC" field, $F_{D C}$, such that $H_{0}$ corresponds here to the Hamiltonian of an atom in a static electric field and, therefore, inducing Stark beats. The time evolution of such wavepacket can be studied by superposing a very short HCP after a given time delay. In the following subsections we analyze in more detail the dynamics of the atoms for the two schemes of Figure 1.

\section{A. Experimental setup}

Our experimental setup has been briefly described elsewhere $[17,10]$. The initial stationary Rydberg atoms are created by photoexciting potassium atoms using the focussed output of an intracavity-doubled CR699-21 dye laser. Excitation occurs near the center of an interaction region defined by three pairs of planar electrodes that are biased to locally reduce stray electric fields to $\lesssim 50 \mu \mathrm{Vcm}^{-1}$. To minimize motional electric fields, the magnetic field is reduced to $\lesssim 20 m G$ by use of $\mu$-metal shields. Measurements are conducted in a pulsed mode. The laser output is formed into a train of pulses of $\sim 4 \mu s$ duration with a pulse repetition frequency of $\sim 10 \mathrm{kHz}$. (The probability that a Rydberg atom is formed during any pulse is small, $\lesssim 0.01$, and data are accumulated following many laser pulses.).

A few hundred $n s$ after the end of the laser pulse the first HCP is applied. The HCPs in the scheme of Fig. 1(a) are created by applying voltage pulses to a circular copper disc. The pulses were produced using an Avtech model AVI - V pulse generator. The output of the generator is split into two pulses of approximately equal amplitude by a matched resistive power divider. One of the resulting pulses is delayed using a length of RG 402 cable and the two pulses are then recombined using a matched power combiner and transported to the HCP electrode by rigid coaxial cable. The individual pulses can be attenuated and/or inverted by connecting fixed broadband attenuators and/or an inverting pulse transformer in series. Since we are able to measure the shape and absolute fields of our pulses, they can be used in the simulations without involving any adjustable parameters. The number and excited-state distribution of Rydberg atoms remaining in the excitation region is determined, after a time delay of 6 
$\mu s$, by selective field ionization (SFI). Measurements in which no HCPs are applied are interspersed at routine intervals during data acquisition to monitor the number of Rydberg atoms initially produced by the laser. The Rydberg atom survival probability is obtained by comparing the Rydberg atom signals observed with and without HCPs present.

The "rectangular" voltage pulse providing the field step in Fig. 1(a) has a duration of several microseconds and a rise time adjustable down to $\sim 10 \mathrm{~ns}$. Because the pulse must be AC coupled (to remove DC offsets present in the output of the pulse generator), its amplitude (and that of the applied field) decreases by $\sim 10 \%$ over a typical measurement period of 500 ns. The probe HCP is superposed on the "DC" pulse using a power combiner. Possible systematic errors associated with uncertainties in the applied field amplitudes are estimated to be $\lesssim \pm 10 \%$.

\section{B. Momentum wavepackets}

Consider the application of an ultrashort HCP (e.g. the pump in Fig. 1(a)) to a stationary Rydberg state $\left|\phi_{i}\right\rangle=\left|n_{i} l_{i} m_{i}\right\rangle$ with well defined spherical quantum numbers $n_{i}, l_{i}, m_{i}$. The resulting coherent wavefunction is

$$
|\Psi(t=0)\rangle=\left|\phi_{i}^{B}\right\rangle=e^{i \Delta \vec{p} \cdot \vec{r}}\left|\phi_{i}\right\rangle
$$

and corresponds to a Galilei boosted initial state shifted in momentum space in $\Delta \vec{p}$. The corresponding expectation values of the energy and momentum are

$$
\begin{aligned}
\langle E\rangle_{t=0} & =\left\langle\phi_{i}^{B}\left|H_{a t}\right| \phi_{i}^{B}\right\rangle=\left\langle\phi_{i}\left|H_{a t}\right| \phi_{i}\right\rangle+\frac{(\Delta p)^{2}}{2}+\left\langle\phi_{i}|\vec{p} \cdot \Delta \vec{p}| \phi_{i}\right\rangle \\
\langle\vec{p}\rangle_{t=0} & =\left\langle\phi_{i}^{B}|\vec{p}| \phi_{i}^{B}\right\rangle=\Delta \vec{p}+\left\langle\phi_{i}|\vec{p}| \phi_{i}\right\rangle=\Delta \vec{p}
\end{aligned}
$$


where $H_{a t}$ is the atomic Hamiltonian and $\vec{r}$ and $\vec{p}$ are the electron position and momentum operators, respectively. Note that $\langle n l m|\vec{p}| n l m\rangle=0$ and, therefore, $\langle E\rangle_{t=0}=\epsilon_{n_{i}}+(\Delta p)^{2} / 2$ where $\epsilon_{n_{i}}$ is the energy of the initial Rydberg state. Classically, the application of a HCP to an electron with momentum $\vec{p}$ and energy $\epsilon_{n}$ changes its energy according to Eq. 2. The classical average of $\Delta E$ over an ensemble of phase-space points representing the initial quantum state $\left|\phi_{i}\right\rangle$ agrees with the quantum mechanical result $\Delta E=(\Delta p)^{2} / 2$.

The electronic wavefunction after the kick can be expanded in terms of field free eigenstates as

$$
|\Psi(t)\rangle \simeq \sum_{n} e^{-i \epsilon_{n} t} \sum_{l}\langle n l m \mid \Psi(0)\rangle|n l m\rangle
$$

and encompasses a broad distribution of angular momentum states, including high- $l$ states. We choose a quantization $z$-axis in the direction of the HCP and, therefore, $m$ is a constant of the motion.

Figure 2 displays the excitation function (or energy distribution) after the first HCP for different Rydberg states with $n_{i}=417$ and well defined angular momenta. Calculations employing the classical trajectory Monte Carlo (CTMC) approach are included for both an ultrashort pulse $\left(T_{p} / T_{n_{i}}=0\right)$ and a pulse of $\sim 2$ ns duration $\left(T_{p} / T_{n_{i}}=0.18\right)$ as used in the experiments. The HCP leads to population of a range of higher $n$ states centered around $n_{f}=\sqrt{-2\langle E\rangle_{t=0}} \simeq 493$ with a width $\Delta n \simeq 20$. Since $1<<\Delta n<<n_{f}$, the convergence of the quantum evolution (Eq. 8) to the classical limit can be treated in detail. The peak in the energy distribution is completely equivalent to the well-known Bethe ridge in atomic collision physics [22]. Remarkably, this structure also exists in the density of bound states after a short HCP. The "period" of a Kepler orbit with the peak energy is given by

$$
T_{n_{f}} \simeq T_{n_{i}}\left[1-\left(\Delta p_{0}\right)^{2}\right]^{-3 / 2}
$$

where $\Delta p_{0}=\left(\Delta p / p_{n_{i}}\right)=n_{i} \Delta p$. The width of the Bethe ridge is determined by the linear term $\vec{p} \cdot \Delta p$ in 
Eq. 2 and depends on the quantum number (and the geometry) of the initial state. This width determines the width $\Delta n$ of the "wavepacket".

The evolution of such a classical wavepacket, i.e. of the phase space density which initially mimics a quantum circular state $\left(n_{i}=417, l_{i}=m_{i}=416\right)$ is illustrated in Fig. 3 . At $t=0$ the wavepacket is well localized in the $(\rho, z)$ plane $\left(\rho=\sqrt{x^{2}+y^{2}}\right)$ and the HCP delivers a kick towards the positive $z$ axis. For $t>0$ the wavepacket approximately follows the trajectory of an electron initially in a circular orbit contained in the $(x, y)$ plane. The spreading of the wavepacket originates in the energy distribution (Fig. 2) which, in turn, results from the initial distribution of eccentricities and orientations used to mimic the initial quantum state. At $t=T_{n_{f}} / 2$ the wavepacket is moving towards the $z<0$ direction $\left(p_{z}<0\right)$. If a second HCP with $\Delta p_{z}>0$ is applied at this time, the linear $\Delta p$ contribution to the energy transfer (Eq. 2) tends to decrease the final binding energy and stabilizes the atom. Similarly, for a second HCP with $\Delta p_{z}>0$ applied at $t=T_{n_{f}}$, the two sequential momentum transfers $\Delta p$ add up leading to an increase of energy and an enhanced ionization probability.

A comparison of the calculated survival probability of $K(417 \mathrm{p})$ as a function of the delay time between two HCPs with our experimental data is shown in Fig. 4. The survival probability oscillates as a function of the delay in between the two HCPs reflecting the time evolution of the wavepacket which, in turn, follows the time development of the expectation value of the $z$ component, $p_{z}$, of momentum of the electron after the first HCP (see $\left\langle p_{z}\right\rangle$ on the right hand side axis). Fig. 4 contains data for the probe HCP parallel and antiparallel to the pump HCP. The oscillations in the two data sets are phase shifted by $\pi$ which is an obvious consequence of the quasi-classical "orbital" motion of the wavepacket. The amplitude of the oscillation decreases steadily with time, however, because a distribution of final states is excited that evolve differently in time leading to dephasing. The experimental data for both configurations agree well with the calculations. Furthermore, calculations for potassium and for hydrogen are found to be indistinguishable. This is due to the fact that the electron in the initial Rydberg state is most of the time away from the $K^{+}$core and that after the first HCP large angular momentum states are populated. 
The very good agreement between theory and experiment demonstrates that classical dynamics can reproduce the "quantum beats" observed in the data and moreover that the classical evolution mimics the quantum evolution. On general grounds [23], one expects the quantum and the classical evolution to depart from each other at a characteristic "break" time $t_{c} \simeq 2 \pi / \Delta \epsilon$, where $\Delta \epsilon$ is the smallest level spacing. For Rydberg states, the level spacing $\epsilon_{n+1}-\epsilon_{n} \simeq n^{-3}$ and $t_{c}$ corresponds to the classical Kepler orbita! period $T_{n}=2 \pi n^{3}$. Remarkably, classical-quantum correspondence extends for times well beyond $t_{c}$. This extended classical-quantum correspondence can be traced to the particular properties of the Coulomb-Kepler problem and of the HCP-generated wavepackets. The energy differences in the Rydberg series determining quantum beats are given to second order in $\delta n / n_{f}$ by

$$
\epsilon_{n_{f}+\delta n_{n}}-\epsilon_{n_{f}} \simeq \delta n \omega_{n_{f}}\left[1-\frac{3}{2} \frac{\delta n}{n_{f}}+2\left(\frac{\delta n}{n_{f}}\right)^{2}\right]
$$

where $|\delta n| \leq \Delta n / 2$. To leading order, the spectrum compares locally to that of a harmonic quantum oscillator, with $\omega_{n_{f}}=2 \pi / T_{n_{f}}$ being the classical orbital frequency. For a harmonic oscillator, quantum and classical expectation values agree [24] which explains the presence in the classical simulation of beats with the mean orbital period. The damping of the beats is caused by the "anharmonic" correction. Dephasing (by $\pi$ ) or damping occurs over a time $t_{D} \simeq 4 n_{f} / 3(\Delta n)^{2} T_{n f} \simeq 2 T_{n_{f}}$ in agreement with the observations. The fact that classical dynamics can accurately reproduce even the dephasing results because, when $\Delta n$ is large, the approximation of a large but discrete set by a continuous distribution is valid. Classical dynamics will fail, however, at times approaching the revival time $t_{R}$ of the wavepacket. Complete revival requires $t_{R} \simeq\left(n_{f} / 3\right) T_{n},[5]$ which, for the values of $n_{f}$ of interest here, is quite long and out of reach of the experiment.

Fig. 5 shows expectation values $\left\langle p_{z}\right\rangle$ calculated using both classical and quantum methods following application of an ultrashort pulse providing a momentum transfer $\Delta p / p_{n_{i}}=0.53$ to hydrogen atoms initially in the $n_{i}=100, l_{i}=0$ state.$n_{i} \approx 100\left(n_{f} \simeq 118\right)$ is currently our upper limit for which the 
necessary matrix elements (Eq. 8) can be computed with sufficient accuracy. The width of the band populated by the HCP is $\Delta n=16$. The classical and quantum results agree up to $\simeq 6 T_{n_{f}}$, i.e. six times the quantum breaktime $t_{c}$. Classical-quantum correspondence breaks down completely for longer times where revivals occur which are absent in classical dynamics. Such revivals have been observed in HCP studies at $n_{i} \sim 25$ [16]. Revival of the wavepacket can be treated semiclassically [5].

\section{Stark wavepackets}

Consider now the application of a field "step" (e.g. the pump in Fig. 1(b)) on a stationary Rydberg state. If the rise time of the "DC" pulse is very short, $|\Psi(0)\rangle \simeq\left|\phi_{i}\right\rangle$ in Eq. 3, and $\epsilon_{\alpha}$ and $\left|\chi_{\alpha}\right\rangle$ are the eigenenergies and eigenvectors of $H_{0}=H_{a t}+z F_{D C}$. In this case, however, only states with a narrow distribution of $n$ values $(\Delta n \lesssim 4)$ centered at $n \simeq n_{i}$ contribute significantly to the summation in Eq. 3 . Expressed in parabolic quantum numbers, the eigenenergies for hydrogen in a DC field are given to first order by

$$
\epsilon_{\alpha}=-\frac{1}{2 n^{2}}+\frac{3}{2} F_{D C} n\left(n_{1}-n_{2}\right)
$$

The relevant energy differences (beating frequencies) governing the time development of the wavepacket are approximately given by

$$
\Delta \epsilon \simeq w_{o r b} \delta n+w_{S} k \quad ; \quad k=0,1, \ldots,\left(n_{i}-|m|-1\right)
$$

The constant energy differences are reminiscent of two harmonic oscillators, one with the classical orbital frequency, $w_{\text {orb }}=n_{i}^{-3}$, and one with the so called Stark frequency, $w_{S}=3 n_{i} F_{D C}$. Classically, a simple quasiperiodic evolution of the wavepacket with frequency $w_{\mathcal{S}}$ is expected to occur corresponding to the precession of the the pseudospins

$$
\vec{j}_{1,2}=\frac{1}{2}\left(\vec{L} \pm \frac{\vec{A}}{\sqrt{-2 H_{a t}}}\right)
$$


with respect to the electric field [25]. In this equation $\vec{L}$ is the angular momentum and $\vec{A}=\vec{p} \times \vec{L}-(\vec{r} / r)$ is the Runge-Lenz vector, which points towards the perihelion of a Kepler orbit. To first order, $\vec{j}_{1,2}$ precede in time according to

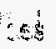

$$
\frac{d \vec{j}_{1,2}}{d t} \simeq \pm \frac{w_{S}}{2} \hat{z} \times \vec{j}_{1,2}
$$

The corresponding geometrical picture of the time evolution is illustrated in Figure 6. The precession of the pseudospins leads to a periodic fluctuation of $\vec{L}$ and ${ }^{x} \vec{A}$ as shown in Fig. 6 (b) (i.e. a periodic fluctuation of the eccentricity and the orientation of the electronic orbit, $\cos (\theta)=A_{z} / A=$ Const. $\left./ A\right)$. At $t=0$ the vectors $\vec{j}_{1}$ and $\vec{j}_{2}$ lie in the $y$-z plane pointing in opposite directions $\left(j_{1_{y}}>0, j_{2_{y}}<0\right)$, $|\vec{L}|$ is at its minimum and $|\vec{A}|$ is at its maximum (i.e. $A^{2}+2\left|H_{a t}\right| L^{2}=1$ ). After half a Stark period, $t=T_{S} / 2=\pi / w_{S}$, the pseudospins lie in the $\mathrm{x}-\mathrm{z}$ plane with negative projections along the $\mathrm{x}$-axis, and $|\vec{L}|$ acquires its maximum value. At $t=T_{S}=2 \pi / w_{S}, \vec{j}_{1}$ and $\vec{j}_{2}$ lie, in the y-z again but with $j_{1_{y}}<0, j_{2_{\nu}}>0$, and $|\vec{L}|$ reaches its second minimum. The quantum evolution of Rydberg atoms in DC fields follows the same periodic pattern [18].

For potassium and the present DC field strengths Eq. 12 breaks down because many Stark manifolds $\mathrm{r}$ overlap and mixing occurs. In this regime, the Stark map for potassium is characterized by a large number of avoided crossings with a nearest neighbor energy level statistics close to a chaotic Wigner distribution. It is therefore not obvious apriori that any simple beat pattern would emerge. We have found, however, that in the high-n limit the weighted quantum statistical distribution of eigenenergies in Eq. 3 is such that a single dominant Stark dominates, closely resembling the result for hydrogen [19].

Data obtained for $\mathrm{K}(388 \mathrm{p})$ with a "DC" field of $\sim 5 \mathrm{mVcm}^{-1}$ and $10 \mathrm{~ns}$ rise time are presented in Fig. 7, which shows the survival probability as a function of the time delay between application of the "DC" and HCP pulses for different HCP amplitudes and polarities. Sizable periodic oscillations (quantum beats) in the survival probability are evident which, measurements show, continue for at least $1.5 \mu \mathrm{s}$. Figure 7 includes results obtained with a "DC" field of $\sim 10 \mathrm{mV} \mathrm{cm}{ }^{-1}$ which demonstrate that 
the quantum beat frequency is proportional to the magnitude of the "DC" field. Results of CTMC calculations reproduce well the observed beats. The small differences in magnitude and period can be attributed to the uncertainty inherent in determining the value of the "DC" and HCP fields. The classical survival probability clearly exhibits the two quite dissimilar Stark, $w_{S}$, and orbital, $w_{\text {orb }}$, frequencies. For $n_{i} \sim 388$ and $F_{D C}=5(10) m V c m^{-1}$, the Stark period $T_{S} \sim 134(67) n s$, is close to the values measured experimentally. The experimental data contain only a hint of the high frequency fluctuations associated with $w_{\text {orb }}$, possibly as a result of small field inhomogeneities in the experimental region.

The positions of the maxima and minima of the survival probability depend on the size and the polarity of the HCP. Using Eq. 2, this can be explained in terms of the time evolution of the $z$ component, $p_{z}$, of the momentum of the excited electron. Figure 8 shows the calculated distribution of the scaled electron momentum, $n_{i} p_{z}$, as a function of time following the application of the "DC" pulse. At early times, the distribution is sharply peaked at small negative values of $p_{z}$. As time advances, the distribution broadens. The peak moves toward more negative values and decreases in size before finally disappearing. A small peak then appears in the distribution at positive values of $p_{z}$ which grows and moves toward $p_{z}=0$ as the distribution narrows. Ultimately the peak crosses to negative values of $p_{z}$, and the whole cycle repeats. If a short HCP is used to probe the complex dynamics of the Stark wavepacket, only. electrons with $\Delta E>-\epsilon_{n_{i}}$ become ionized. Using Eq. 2, this implies $p_{z} \Delta p_{z}>-\epsilon_{n_{i}}-\left(\left(\Delta p_{z}\right)^{2} / 2\right)$ which is equivalent to making a cut of the momentum distribution in Figure 8. The result of this cut depends on the, strength and the direction of the HCP through the linear term in $\Delta p_{z}$.

\section{CONCLUSIONS}

In summary, the present work demonstrates that very-high-n Rydberg wavepackets can be created using HCPs. The motion of the wavepackets is quasiperiodic with periods that can be directly measured using a probe HCP. The resulting survival probability of atoms exhibits pronounced oscillations (quantum beats) that persist for a long period of time. The very good agreement between classical results and experiment 
demonstrates that, for the range of times studied experimentally, classical-quantum correspondence holds. This is a consequence of the fact that in the limit of large principal quantum numbers energy levels become nearly equidistant.

Since the first order Stark energy levels are equidistant, very little dephasing is observed in the Stark beats. Only a very small amount of dephasing has been found to occur due the finite size of the $K^{+}$core. This suggests that it might be possible to observe external damping induced by dephasing in collisions with a target gas. If so, such studies would provide a new tool for investigating elastic electron-molecule scattering at electron energies down to a few microelectronvolts.

\section{ACKNOWLEDGEMENTS}

The experimental work was supported by the NSF and the Robert A. Welch Foundation. The theoretical work was funded by the U. S. DoE, OBES, Div. of Chem. Sciences, under Contract No. DE-AC05-96OR22464 with ORNL managed by LMERC and by the NSF. It is a pleasure to acknowledge the assistance of $\mathbf{B}$. Tannian in data acquisition. 


\section{References}

[1] E. Schrödinger, Naturwissenschaften 14, 664 (1926).

[2] B.H. Bransden and M.R.C. McDowell 1992 Charge Exchange and the Theory of Ion-Atom Collisions (Oxford University Press, Oxford).

[3] J. Andrä, Phys Rev A 2, 2200 (1970); I.A. Sellin, J.R. Mowat, R.S. Peterson, P.M. Griffin, R. Laubert, and H.H. Haselton, Phys. Rev. Lett. 31, 1335 (1973); J. Burgdörfer, Phys.Rev. A 24, 1956 (1981).

[4] K.B. MacAdam, J.C. Day, J.C. Aguilar, D.M. Homan, A.D. MacKellar, and M.J. Cavagnero, Phys.Rev.Lett. 75, 1723 (1995); D.R. Schultz, C.O. Reinhold, and P. Krstic, Phys.Rev.Lett. 78, 2720 (1997).

[5] J. A. Yeazell and C. R. Stroud, Phys. Rev. Lett. 60, 1494 (1988). M. Mallalieu and C. R. Stroud, Phys. Rev. A 49, 2329 (1994).

[6] A. ten Wolde, L. D. Noordam, A. Lagendijk, and der Zande, H. B. van Linden van den Heuvell and, Phys. Rev. Lett. 61, 2099 (1988); B. Broers, J. F. Christian, J. H. Hoogenraad, W. J. van der Zande, H. B. van Linden van den Heuvell and L. D. Noordam, Phys. Rev. Lett. 71, 344 (1993).

[7] R. Bluhm, V.A. Kostelecky, J.A. Porter, Am. J. Phys. 64, 944 (1996).

[8] C. Raman, C. W. S Conover, C. I. Sukenik, and P. H. Bucksbaum, Phys. Rev. Lett. 76, 2436 (1996).

[9] R. R. Jones, D. You, and P. H. Bucksbaum, Phys. Rev. Lett. 70, 1236 (1993).

[10] M. T. Frey, F. B. Dunning, C. O. Reinhold, and J. Burgdörfer, Phys. Rev. A., 53, R2929 (1996).

[11] C. O. Reinhold, M. Melles, and J. Burgdörfer, Phys. Rev. Lett. 70, 4026 (1993); C. O. Reinhold, M. Melles, H. Shao, and J. Burgdörfer, J. Phys. B 26, L659 (1993); 29, 377 (1996).

[12] C. O. Reinhold and J. Burgdörfer, Phys. Rev. A 51, R3410 (1995); C. O. Reinhold, J. Burgdörfer, R. Jones, C. Raman, P. Bucksbaum, J. Phys. B 28, L457 (1995).

[13] P. Krstic and Y. Hahn, Phys. Rev. A 50, 4629 (1994); C. D. Schwieters and J. B. Delos, Phys. Rev. A 51, 1023 (1995); A. Bugacov, B. Piraux, M. Pont, and R. Shakeshaft, Phys. Rev. A 51, 1490 (1995); K. J. LaGattuta, and P. Lerner, Phys. Rev. A 49, R1547 (1994); V. Enss, V. Kostrykin, and R. Schrader, Phys. Rev. A 50, 1578 (1994).

[14] N. E. Tielking, T. J. Bensky, and R. R. Jones, Phys. Rev. A 51, 3370 (1995); N. E. Tielking and R. R. Jones, Phys. Rev. A 52, 1371 (1995).

[15] G. M. Lankhuijzen and L. D. Noordam 1994 Phys. Rev. Lett. 74, 35; P. Kristensen, G. M. Lankhuijzen and L. D. Noordam, J. Phys B 30, 1481 (1997).

[16] R. R. Jones, Phys. Rev. Lett. 76, 3927 (1996).

[17] M. T. Frey, S. B. Hill, K. A. Smith, and F. B. Dunning, Phys. Rev. Lett. 75, 810 (1995).

[18] C. O. Reinhold; H. Shao and J. Burgdörfer, J Phys B 27 (1994) L469.

[19] M. T. Frey, F. B. Dunning, C. O. Reinhold, and J. Burgdörfer, Phys. Rev. A., 55, R865 (1997).

[20] C. O. Reinhold, J. Burgdörfer, M. T. Frey, and F. B. Dunning, Phys. Rev. A., 54, R33 (1996).

[21] J. Burgdörfer, and J. Gibbons, Phys. Rev. A 42 (1990) 1206; J. Kemmler, J. Burgdörfer, and C.O. Reinhold, Phys. Rev. A 44, 2993 (1991).

[22] M. Inokuti, Rev. Mod. Phys. 43, 297 (1971); C.O. Reinhold and J. Burgdörfer, J. Phys. B 26, 3101 (1993).

[23] M. Berry, Physica 33D, 261 (1988).

[24] A. Messiah, Quantum Mechanics Vol. 1 (North-Holland, Amsterdam, 1967).

[25] M. Born, Vorlesungen Über Atommechanik, (Springer, Berlin 1926). The Mechanics of the Atom, (G. Bell and Sons, London 1926). 


\section{Figure Captions}

Fig. 1. Experimental pulse profiles used to produce and probe Rydberg wavepackets: (a) the pump and the probe are given by short HCPs separated by a time delay; (b) the pump and the probe are given by a field step and a short HCP, respectively.

Fig. 2. Probability density to find the electron with a given binding energy following the application of: an ustrashort HCP with $\Delta p_{0}=\Delta p / p_{n_{i}}=0.53$ to $\mathrm{H}(417 \mathrm{p})$ and $\mathrm{H}\left(n_{i}=417, l_{i}=m_{i}=416\right)$ and a pulse of $2 \mathrm{~ns}$ duration $\left(T_{p} / T_{n_{i}}=0.18\right)$ on $\mathrm{K}(417 p)$. The energy axis is scaled to the energy of the initial state, i.e., $E_{0}=E /\left|\epsilon_{n_{i}}\right|$.

Fig. 3. Time evolution of a circular state, $\mathrm{H}\left(n_{i}=417, l_{i}=m_{i}=416\right)$, which at $t=0$ is subject to an ultrashort HCP with $\Delta p_{0}=0.53$. The dots represent a scatter plot of the probability density of the wavepacket. The thick solid line is the trajectory followed by an electron in a perfectly circular orbit initially in the $(x, y)$ plane. The cylindrical coordinates of the electron are scaled to the initial orbital radius: i.e., $z_{0}=z / n_{i}^{2}, \rho_{0}=\rho / n_{i}^{2}$

Fig. 4. Rydberg atom survival probability (left scale) following application of two HCPs in (a) the same and (b) opposite senses to $\mathrm{K}(417 \mathrm{p})$ atoms as a function of time delay. symbols, experimental data; solid lines, results of CTMC calculations. Multiples of $T_{n}$, are marked by arrows. The insets show the pulse profiles. dash line (right scale), time development of the scaled expectation value $\left\langle p_{z}\right\rangle / p_{n_{i}}=n_{i}\left\langle p_{z}\right\rangle$ following application of the first HCP. From Ref. [20]

Fig. 5. Short (a) and long (b) time development (expressed in units of $T_{n_{f}}$ ) of the scaled expectation value $\left\langle p_{z}\right\rangle / p_{n_{i}}=n_{i}\left\langle p_{z}\right\rangle$ following application of a HCP to hydrogen atoms in the $100 s$ state. The scaled momentum transfer $\Delta p / p_{n_{i}}=0.53$. From Ref. [20].

Fig. 6. Stark precesion of (a) the classical pseudospins $\vec{j}_{1,2}$ and (b) the Coulomb orbit about the electric field.

Fig. 7. Rydberg atom survival probability as a function of time delay between application of the "DC" and half-cycle pulses for "DC" fields with a 10 ns rise time and amplitudes of a), b) $5 \mathrm{mVcm}^{-1}$ and c) $10 \mathrm{mV} \mathrm{cm}^{-1}$. In a) the "DC and HCP fields are in the same direction, in b), c) opposite directions. solid circles, open circles, open triangles, experimental data obtained with HCP amplitudes of $\sim 80$, $\sim 170$, and $\sim 290 \mathrm{mVcm}^{-1}$ respectively: solid lines, results of the CTMC calculations. From Ref. [19].

Fig. 8. Time evolution of the probability density of the $z$ component, $p_{z}$, of the electron momentum, plotted as $n_{i} p_{z}$ in a field step of $(5 \mathrm{mV} / \mathrm{cm})$. From Ref. [19]. 


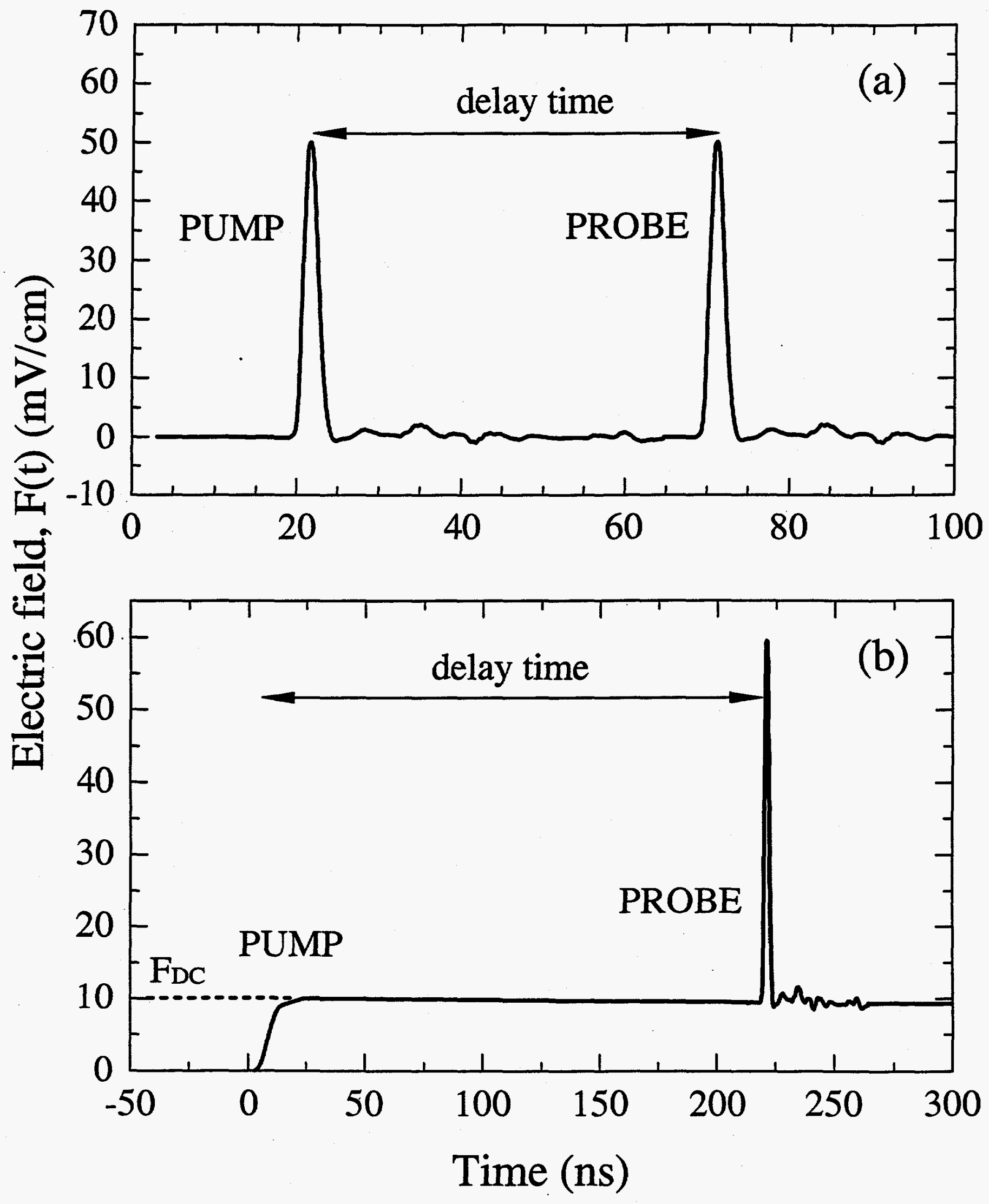

Fig. 1 


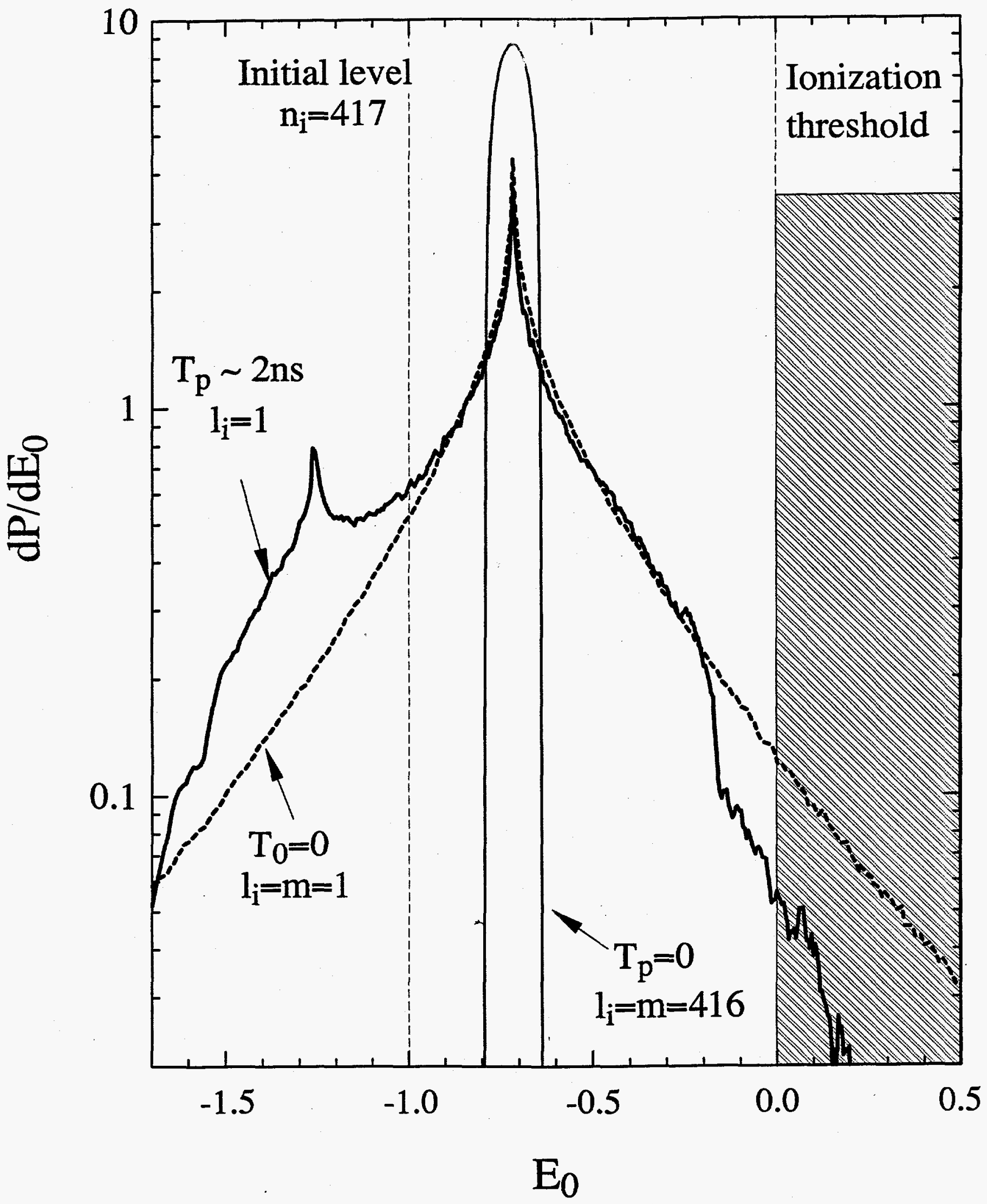

Fig. 2 

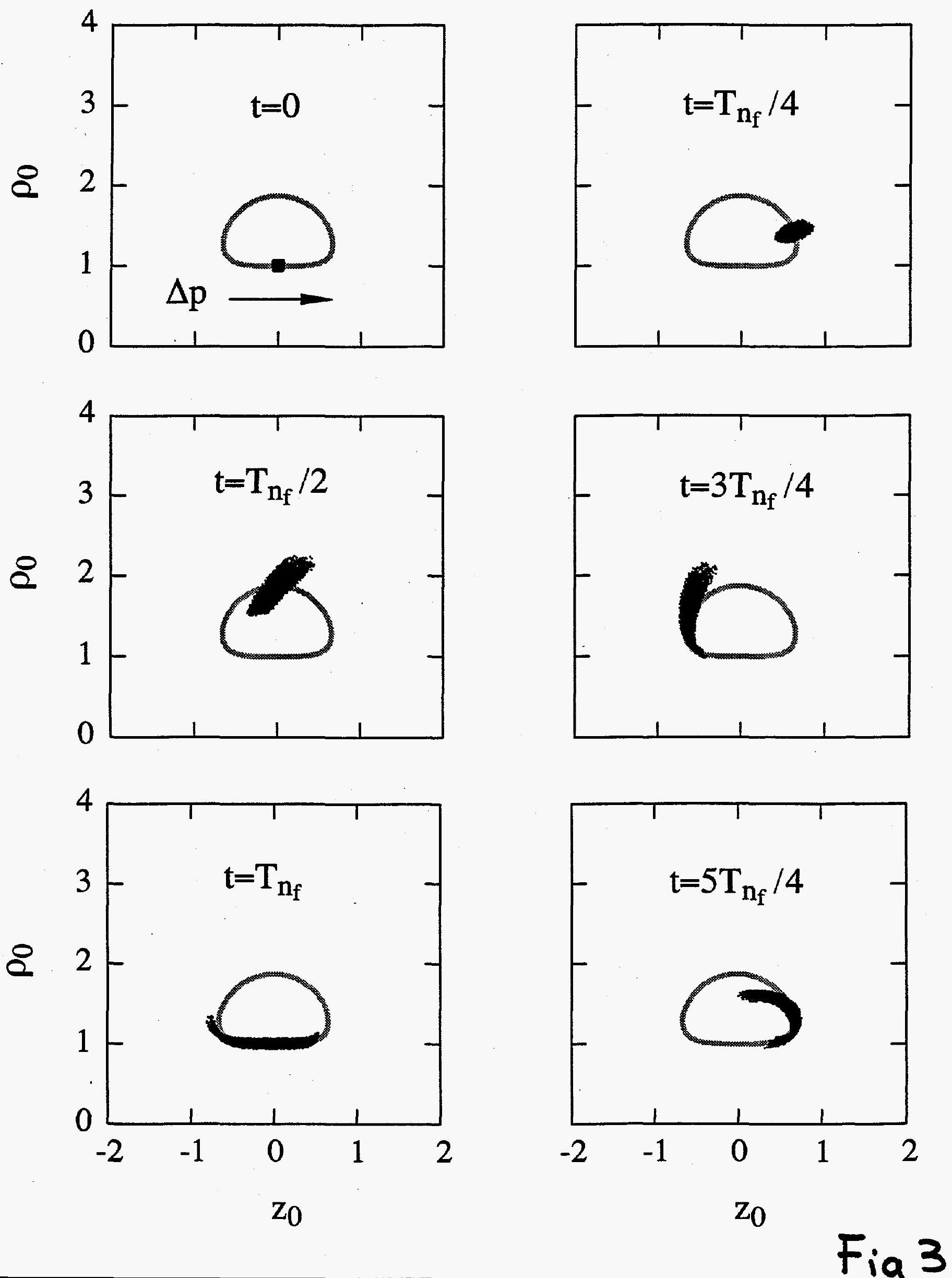


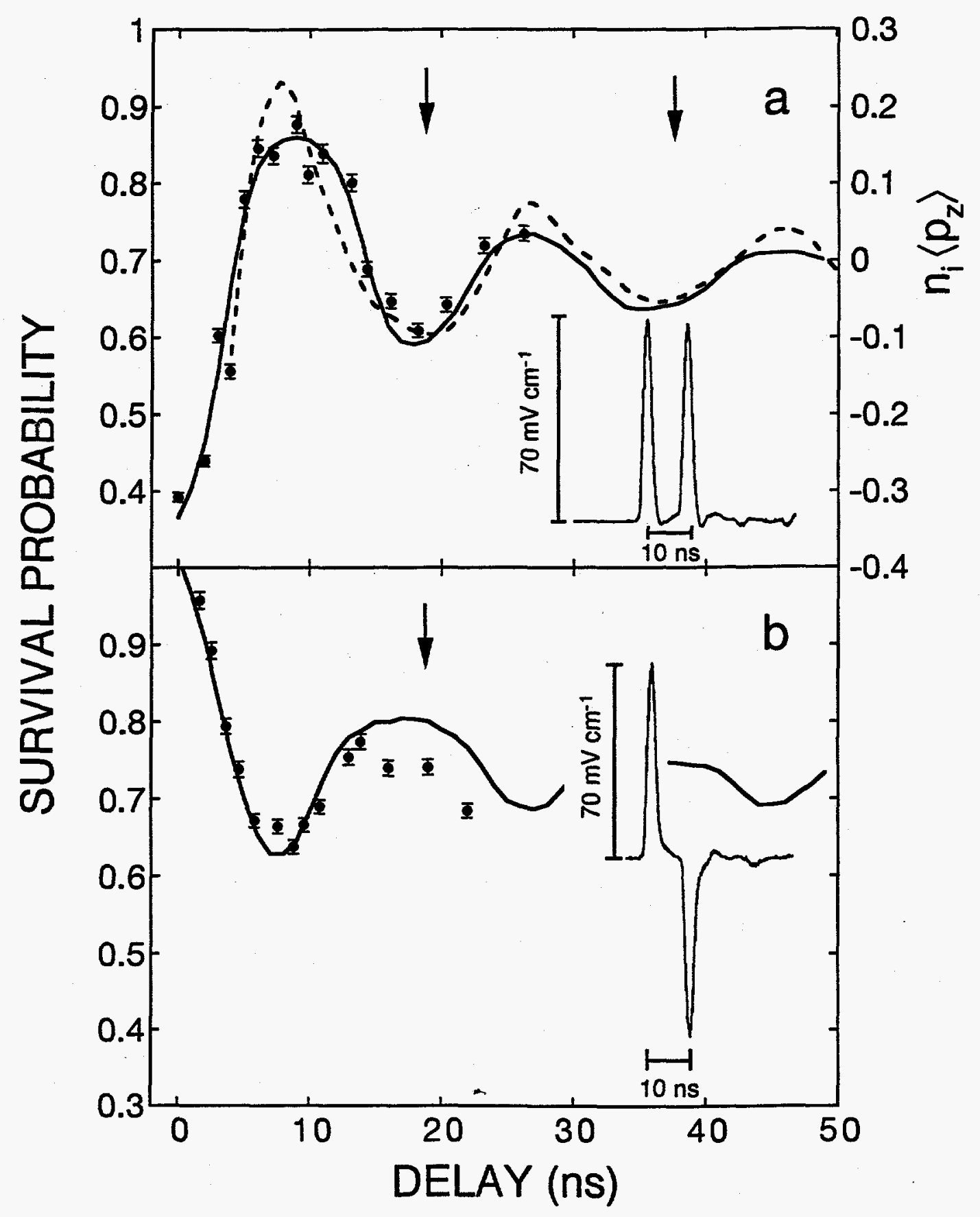

Fig. 4 

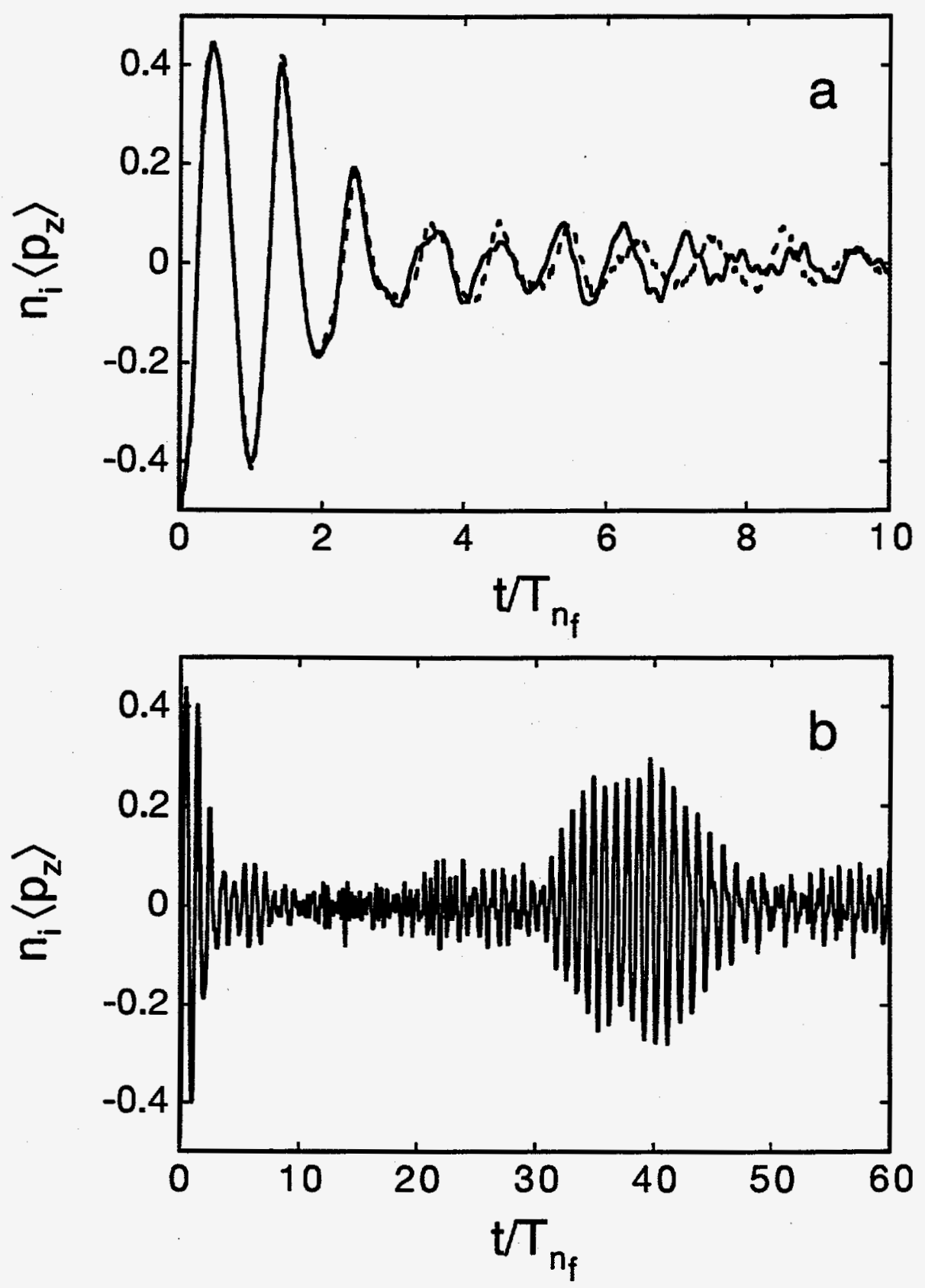

$F_{i g} 5$ 


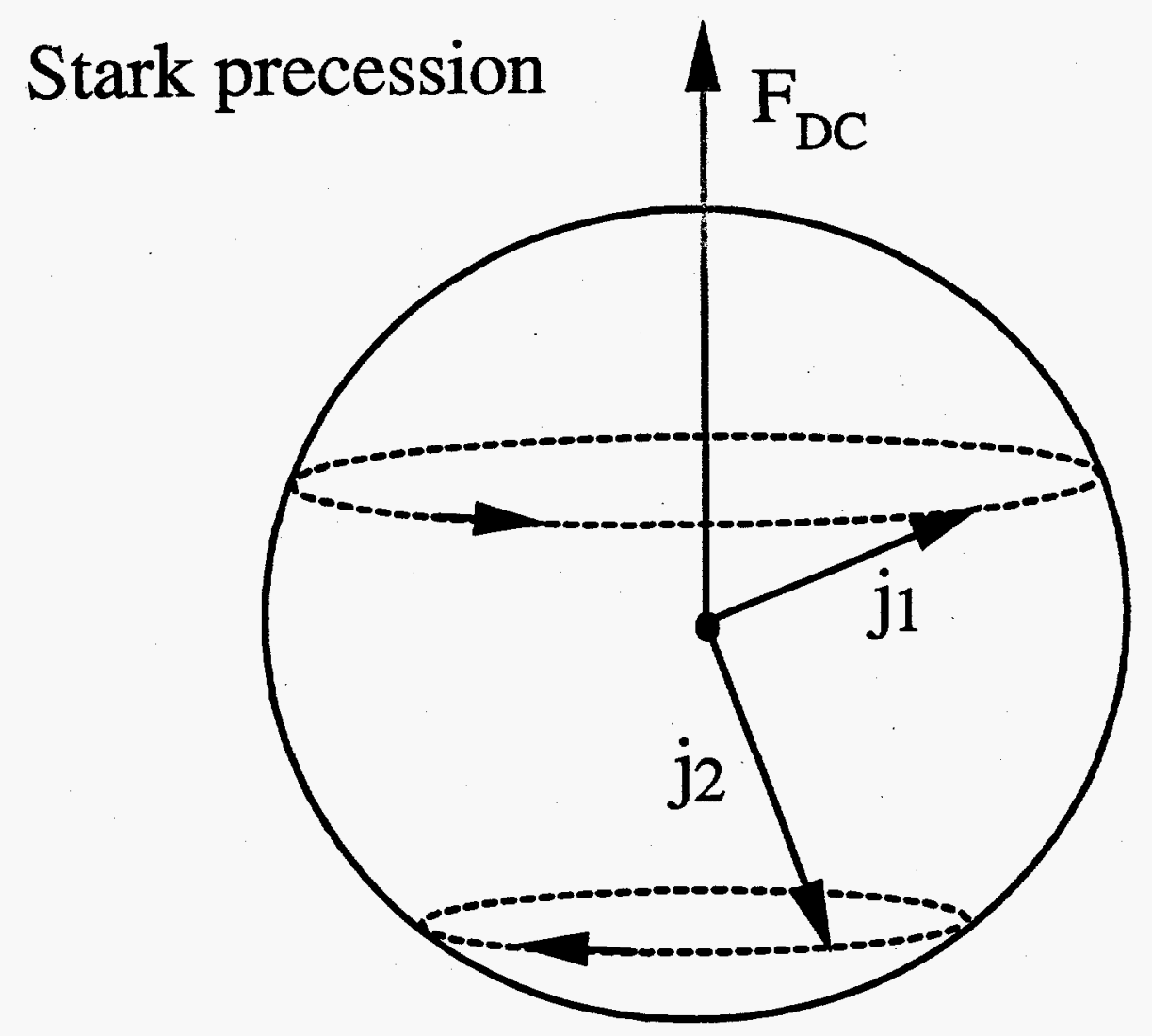

(a)

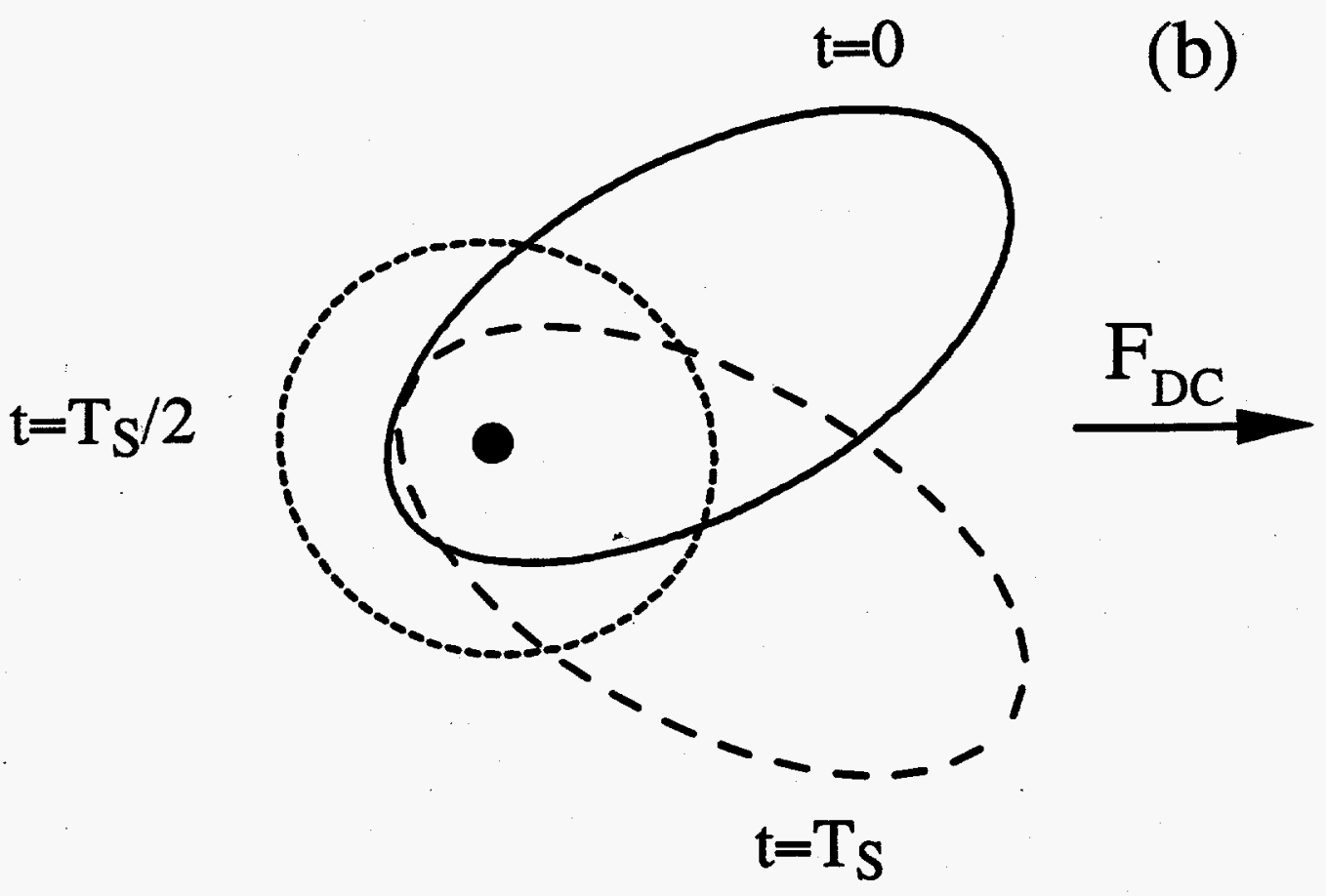

Fig. 6 


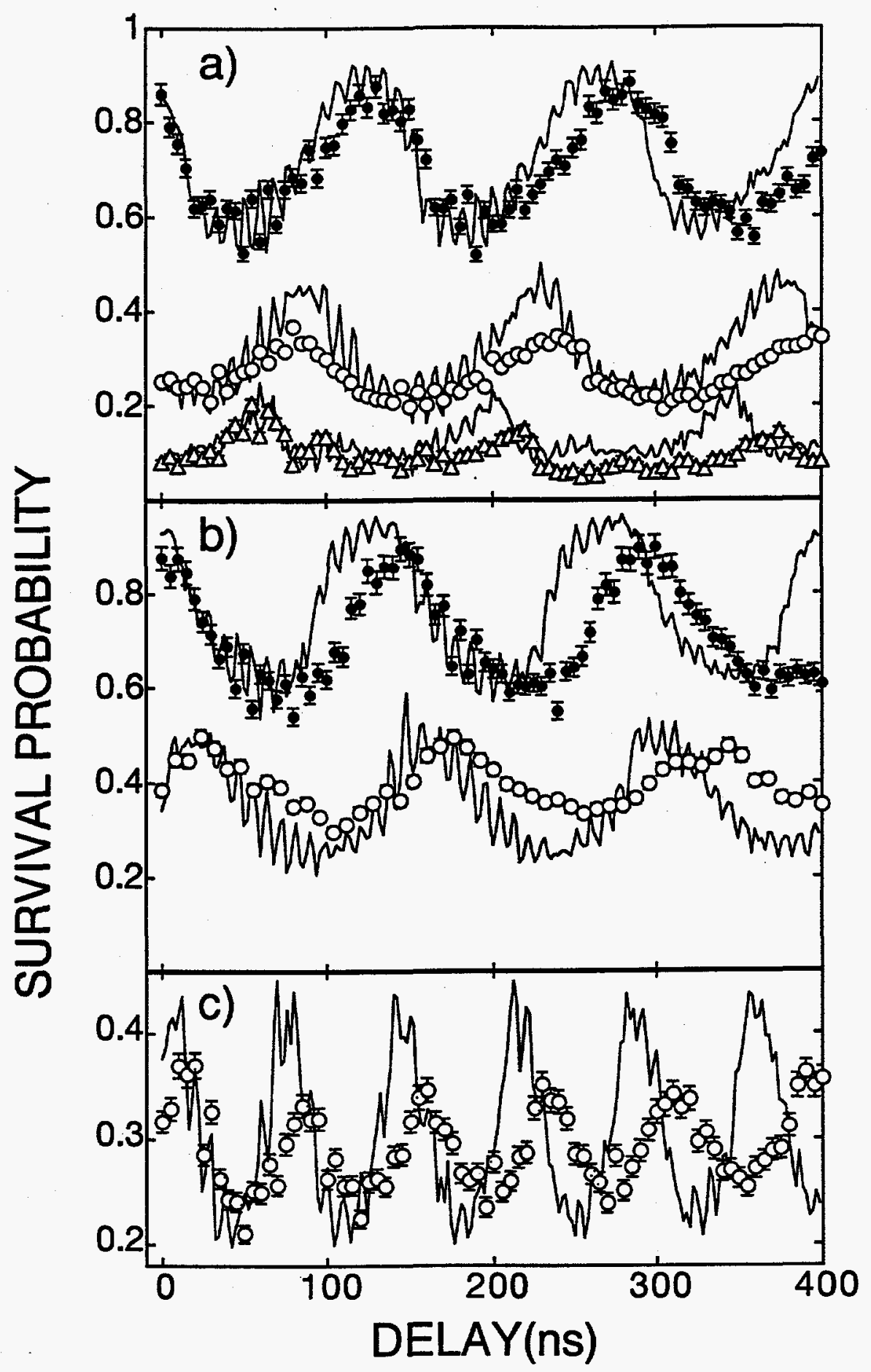

$F_{i g} .7$ 


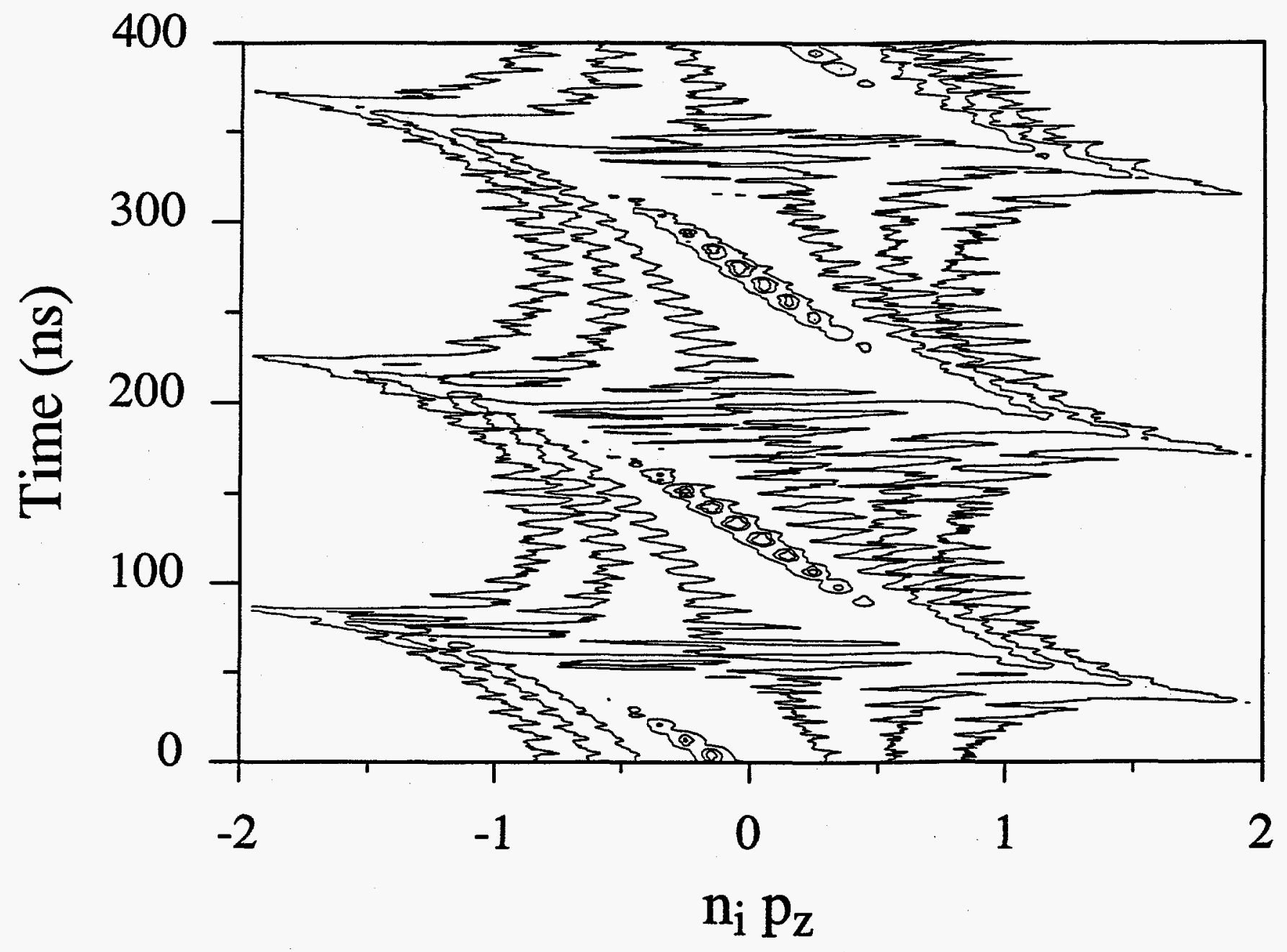

$F_{i g .8}$ 